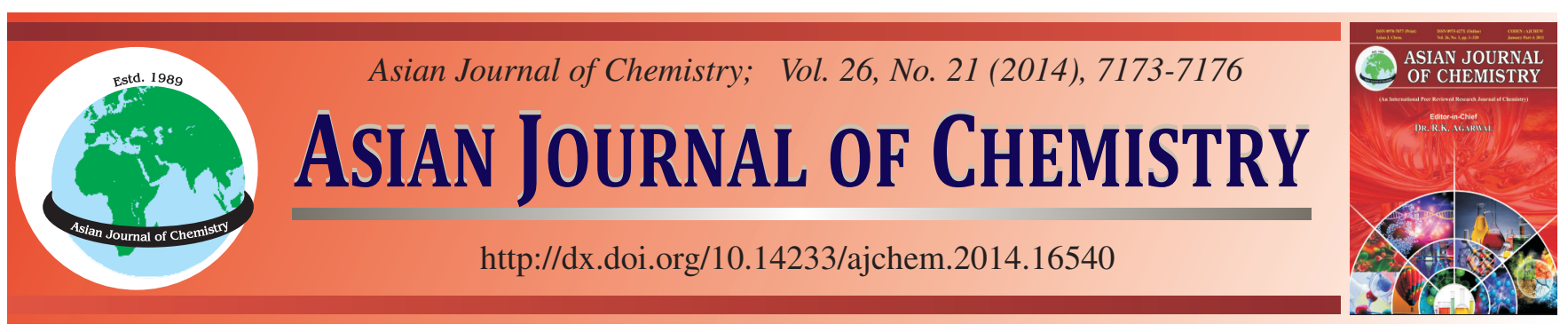

\title{
RP-HPLC Determination of Taxol and 10-Deacetylbaccatin III in Different Harvesting Time of Taxus chinensis Var. Mairei from China
}

\author{
Li-Ping Zhu ${ }^{1}$, Hong-TaO Yang ${ }^{2}$, Yun-Fang Guan ${ }^{2}$ and Ke YuAN ${ }^{1, *}$
}

${ }^{1}$ The Nurturing Station for the State Key Laboratory of Subtropical Silviculture, Zhejiang Agriculture and Forestry University, Lin' an 311300, P.R. China ${ }^{2}$ The Traditional Chinese Medicine Processed Samples of Co. Ltd. in East China of Hangzhou, Lin' an 311300, P.R. China

*Corresponding author: E-mail: yuan_ke001@163.com

Accepted: 24 March 2014;

Published online: 30 September 2014;

AJC-16096

\begin{abstract}
Taxus chinensis var. mairei is well known as anticancer plant. Its main anticancer compounds taxol and 10-deacetylbaccatin III and other diterpenoids have a strong anticancer effects, on clinical practice for the treatment of advanced breast cancer, ovarian cancer and other cancers, has a significant effect. This study established a method for the determination of taxol and 10-deacetylbaccatin III of Taxus chinensis var. mairei and to carry out an experiment for the quantitative determination of taxol and 10-deacetylbaccatin III of branches and leaves in different harvesting time of Taxus chinensis var. mairei. An HPLC method was adopted with Sunfire ODS C18 column $(250 \times 4.6 \mathrm{~mm}, 5 \mu \mathrm{m})$ with the mobile phase consisting of water-acetonitrile (A-B) by gradient program ( $0-20 \mathrm{~min}, 30 \% \mathrm{~A} ; 20-35 \mathrm{~min}$, $30 \% \mathrm{~A} \rightarrow 55 \% \mathrm{~A}, 35-43 \mathrm{~min}, 55 \% \mathrm{~A})$. The flow rate was $1.0 \mathrm{~mL} \mathrm{~min}{ }^{-1}$ with the column temperature was $30{ }^{\circ} \mathrm{C}$ and $\mathrm{UV}$ detection wavelength at $232 \mathrm{~nm}$. The linear range of taxol was $0.020-0.400 \mu \mathrm{g}(\mathrm{r}=0.9993)$ with the average recovery of $100.49 \%$, the linear range of 10 -DAB III was $0.010-0.200 \mu \mathrm{g}(\mathrm{r}=0.9995)$ with the average recovery of $99.65 \%$. The content of the order of two active ingredients of different growth years of Taxus chinensis var. mairei is: 3 years $>4$ years $>2$ years $>1.5$ years $>5$ years. In different harvesting period, the highest content of taxol and 10-deacetylbaccatin III of Taxus chinensis var. mairei is harvested in May and June. The method is simple, accurate and reproducible, and it is applicable for the quality control of Taxus chinensis var. mairei.
\end{abstract}

Keywords: RP-HPLC, Taxus chinensis var. mairei, Taxol, 10-Deacetylbaccatin III.

\section{INTRODUCTION}

Taxus mairei belongs to Taxus genus of Taxaceae also known as yew or the red building has eleven species in the world $^{1}$. There are six species in China, just like Taxus madia, Taxus yunnanensis, Taxus cuspidate, Taxus chinensis var. mairei., Taxus wallichiana Zucc and Taxus chinensis ${ }^{2,3}$. The Taxus genus is the taxol's raw material which is a new developed anticancer medicine in recent years. Taxus chinensis contains a variety of taxane analogues, including more than 10 kinds of composition with antitumor activity ${ }^{4}$.

Taxus chinensis var. maire ${ }^{5}$ also named the beautifulTaxus mairei belongs to the species of Coniferae and Taxaceae as well as Taxus genus ${ }^{6}$. It was called the national key protected wild plant which approved by the State Council on August 4,1999. The distribution of the Taxus chinensis var. mairei mainly in the South of the Yangtze river basin in China, in the forest which about $1000-2000 \mathrm{~m}$ above the sea level ${ }^{7}$. Since the 1970s American scientist get the taxol of anticancer drug from the Taxus brervifolia. Plants of the genus taxus chinensis have been paid much attention by people ${ }^{8-9}$. Nearly forty years Taxus chinensis var. mairei has become a research hotspot in the field of natural drugs in the world. Now Taxus chinensis var. mairei is planting on large areas of China, in order to replace the wild species.

Taxol is one of the three international achievements of antitumor medicine, which was detached from the tree bark of the Taxus chinensis in the pacific and was allowed to come into the market in America in $1992^{10,11}$. The ability of resisting the cancer is higher than that of cis-platinum, etoposide, adriamycin. It has obvious inhibition effect on the breast cancer, the lung cancer, ovarian cancer, mesothelioma. Therefore, it becomes the most effective antitumor medicine ${ }^{12-14}$. At present, the majority of the taxol are obtained from the plants like Taxus chinensis. However, because the taxol only take up a little part in the plants and the resource of natural Taxus chinensisis limited, people rely on other ways to get taxol for many years so that the problem of insufficient of taxol can be solved.

10-Deacetylbaccatin III belongs to taxanes that coexists with taxol in the Taxus chinensis var. mairei. They not only have certain anticancer activity, but can also be modifying the structure to synthesis the taxol and new taxol analogues and to get the taxol drug of efficient and low toxicity of the second 
generation, all of those alleviate the pressure of taxol in short supply on the market in a certain extent ${ }^{15}$.

The RP-HPLC method established in this paper measures simultaneously the contents of taxol and 10-deacetylbaccatin III of Taxus chinensis var. mairei in different harvesting time from China and exploring the change of taxol and 10-deacetylbaccatin III contents, in order to provide a reference for how to use this medicinal resources in much smarter ways.

\section{EXPERIMENTAL}

The HPLC system consisted of a waters 2695-2996 system (Waters Corp., MA, USA), equipped with a binary solvent manager, an auto-sampler and a waters 2996 diode array detector (DAD), was used for liquid chromatographic analysis, the Milli-Q super-purified water device; Shumei KQ2200DE ultrasonic cleaning instrument was used for extraction, AUTO SCIENCE solvent filtration device.

Taxol and 10-deacetylbaccatin III standards were provided by Xian Guanyu Bio-Tech Co., Ltd. ( the purity is over $98 \%$ ). The HPLC grade acetonitrile were purchased from Tedia (Tedia Co., OH, USA), Deionized water was purified by a Milli-Q system from Millipore (Bedford, MA, USA). All the other chemicals and solvents used in sample preparation were of analytical grade.

This experiment used samples of Taxus chinensis var. mairei were collected from the planting base of Taxus chinensis var. mairei of Cixi in Zhejiang Province, China. Those samples were identified by Professor Lu-Huan Lou of Zhejiang Agricultural and Forestry University. The collected samples were dried at $50{ }^{\circ} \mathrm{C}$ and then smash into powder by 60 mesh sieves.

\section{RESULTS AND DISCUSSION}

HPLC chromatographic conditions: An HPLC method was adopted with Sunfire ODS C18 column $(250 \times 4.6 \mathrm{~mm}$, $5 \mu \mathrm{m})$ with the mobile phase consisting of water- acetonitrile (A-B) by gradient program ( 0-20 min, 30\% A; 20-35 min, $30 \% \mathrm{~A} \rightarrow 55 \% \mathrm{~A}, 35-43 \mathrm{~min}, 55 \% \mathrm{~A})$. The flow rate was $1 \mathrm{~mL} \mathrm{~min}^{-1}$ with the column temperature was $30{ }^{\circ} \mathrm{C}$ and UV detection wavelength at $232 \mathrm{~nm}$. The injection volume was 1.0 $\mu \mathrm{L}$. The separation between the chromatographic peak of adjacent degrees were greater than 1.5 , number of theoretical pedal $>30000$. Chromatograph chart as shown in Fig. 1

Preparation of standard solution: Precision weigh taxol reference substance $5 \mathrm{mg}$, dissolved it to volumetric flasks in $25 \mathrm{~mL}$ methanol, shake well, get the taxol standard solution $\left(0.20 \mathrm{mg} \mathrm{min}^{-1}\right)$. Precision weigh 10 -deacetylbaccatin III reference substance $5 \mathrm{mg}$, put it in the $25 \mathrm{~mL}$ volumetric flasks with added methanol for obtaining the 10-deacetylbaccatin III standard solution $\left(0.10 \mathrm{mg} \mathrm{min}^{-1}\right)$. Accurately weigh $1 \mathrm{~mL}$ of the taxol standard solution $\left(0.20 \mathrm{mg} \mathrm{min}^{-1}\right)$ and $1 \mathrm{~mL}$ of the 10-deacetylbaccatin III standard solution $\left(0.10 \mathrm{mg} \mathrm{min}^{-1}\right)$

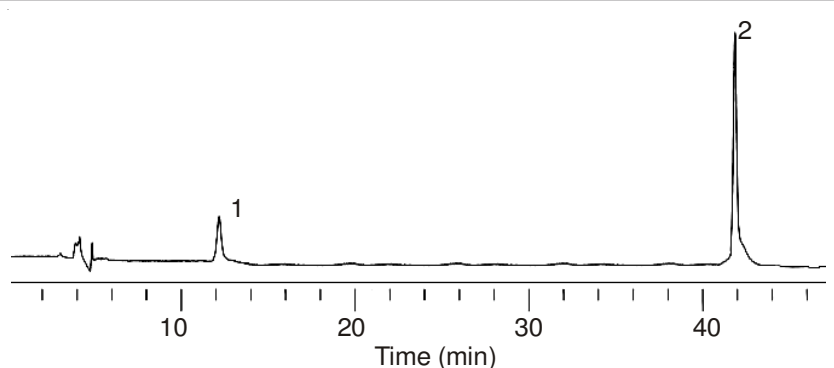

(a)

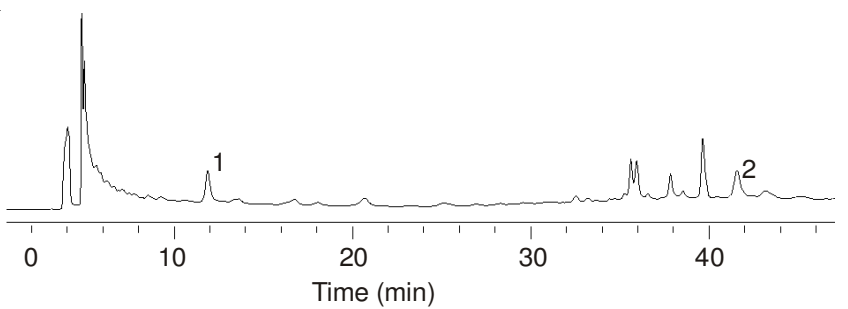

(b)

Fig. 1. Chromatogram of reference substances (a) and sample (b). 1 (10deacetyl-bacratin III) 2 Taxol

respectively and put them in $10 \mathrm{~mL}$ volumetric flask and then resolve them by using methanol and regard them as the mixed standard solution. Avoid light kept at low temperature and to use it right after it was ready.

Preparation of sample solution: The collected samples of Taxus chinensis var. mairei were accurately weighed (approximately $1.0 \mathrm{~g}$ ) and ultrasonic-extracted $0.5 \mathrm{~h}$ with $50 \mathrm{~mL}$ of methanol for three times, the extracts of the collected samples of Taxus chinensis var. mairei were concentrated into about $18 \mathrm{~mL}$ at $45^{\circ} \mathrm{C}$ by the rotary evaporator, then added $2 \mathrm{~mL}$ distilled water, after mixed, added $30 \mathrm{~mL}$ of petroleum ether extracting for 3 to 4 times until the petroleum ether layer was nearly colourless. The methanol phase were evaporated to remove methanol, then added $30 \mathrm{~mL}$ methylene chloride and $15 \mathrm{~mL}$ distilled water extraction. The water layer extraction two times again by $20 \mathrm{~mL}$ of methylene chloride, the methylene chloride extraction that has been combined was evaporated to dryness under reduced pressure, next, dissolved them to a volumetric flasks use $25 \mathrm{~mL}$ methanol. then filtered it through $0.45 \mu \mathrm{m}$ filtration membrane, get the sample solution.

Calibration curve drawing: The mixed reference solution injected for analysis purposes according to the chromatographic conditions, the sampling volume were 1, 3, 5, 7, 10, $15,20 \mathrm{~mL}$ and record the peak area. As a result, the standard curves were obtained by plotting peak area (y) $v s$. the content of authentic standards (x (ug)) of each compound and were fitted to linear regression $y=a x+b$ in Table- 1 .

Precision experiment: Taking the mixed reference fluid and continually injecting for 5 times, the result is the RSD of taxol and 10-deacetylbaccatin III are $1.58 \%$ and $1.13 \%$ respectively, which shows a high accuracy.

TABLE-1

CALIBRATION CURVES AND CORRELATION COEFFICIENTS OF TWO COMPONENTS IN THE MIXED REFERENCE FLUID $(\mathrm{n}=6)$

\begin{tabular}{cccc}
\hline Components & Regression equation & r & Linear range $(\mu g)$ \\
\hline Taxol & $\mathrm{Y}=1.70 \times 10^{6} \mathrm{X}-3.78 \times 10^{4}$ & 0.9993 & $0.020-0.400$ \\
10 -DAB III & $\mathrm{Y}=6.40 \times 10^{5} \mathrm{X}+4.06 \times 10^{2}$ & 0.9995 & $0.010-0.200$ \\
\hline
\end{tabular}


Stability of the samples: Taking the same sample solution and injecting at different time $0,2,4,6,8,10,12 \mathrm{~h}$, we found that the RSD of the peak area of the taxol and 10-deacetylbaccatin III are $1.98 \%$ and $1.26 \%$, respectively, which shows a good stability within $12 \mathrm{~h}$.

Reproducibility: Precision weigh the same medicinal materials of Taxus chinensis var. mairei, the reproducibility was assessed by analyzing the same medicinal materials of Taxus chinensis var. mairei, using the above method. The RSD of the peak area of the taxol and 10-deacetylbaccatin III are $1.52 \%$ and $1.23 \%$, respectively. Thus, the result indicated that the method shows a good reproducibility

Recoveries: Precision weigh the Taxus chinensis var. mairei that the content of the taxol and 10-deacetylbaccatin III is known 6 copies and each has $1 \mathrm{~g}$ with precision, then adding a certain amount of taxol and 10-deacetylbaccatin III reference solution, after that, according to the preparation method of sample solution and the above chromatography conditions we determine the samples content. The results showed that the average recoveries of taxol and 10-deacetylbaccatin III were $100.49 \%$ and $99.65 \%$, respectively.

Determination of the content of the sample: The Taxus chinensis var. mairei of different growth years and different harvesting period were accurately weighed $(1.0 \mathrm{~g})$ respectively. Then use the preparation method of sample solution and the above chromatography conditions we determine the samples content, the result of the content of two components in Taxus chinensis var. mairei were shown in Tables 2 and 3.

Selection of detection wavelength: We found the wavelength of maximum absorption of taxol is $227 \mathrm{~nm}$, while the wavelength of maximum absorption of 10-deacetylbaccatin III is $232 \mathrm{~nm}$ by UV spectroscopy scanning. In order to facilitate testing, finally we chose $232 \mathrm{~nm}$ as the monitoring wavelength.

Selection of moving phase: Taxol and 10-deacetylbaccatin III easily dissolved in acetonitrile-water system, so choose the system as mobile phase, by changing the proportion of aceto- nitrile, we could make the target peak and the impurity peak completely separated from each other.

Selection of extracting conditions: Through observing and studying the extraction effects of different extraction methods, different extraction solvent and extraction times on taxol and 10-deacetylbaccatin III, we found that under the extraction method of at $45^{\circ} \mathrm{C}$ and ultrasonic extracted for three times, $0.5 \mathrm{~h}$ per time, moreover the dosage of methanol is in turn 50, 30 and 20 times of sample volume, the taxol and 10deacetylbaccatin III of Taxus chinensis var. mairei could be fast and efficient extracted completely.

Inspection of extraction solution of methylene chloride: Observing and studying the extraction volume (10, 20, $30 \mathrm{~mL})$ and extraction number of times (1, 2, 3, 4 times), we found that a small amount of extraction can save the solvent and make the target component extracted completely, so we finally decided to use $30 \mathrm{~mL}$ methylene chloride extraction 1 time first, then use $20 \mathrm{~mL}$ methylene chloride extraction 2 times.

The analysis results showed that in the different growth years of Taxus chinensis var. mairei, the highest contents of taxol and 10-deacetylbaccatin III of the medicinal materials is in the third year, which means the effective composition contents of 3-yearold Taxus chinensis var. mairei is higher than others. Compared with other harvesting period of 3-year-old Taxus chinensis var. mairei, the highest content of effective elements of the medicinal materials is in May and June. Thus we predicted the the best harvesting time of Taxus chinensis var. mairei is in May and June. Based on the results and our comprehensive consideration, we predicted that May and June may be the best collection time of 3-year-old Taxus chinensis var. mairei.

This experiment adopts the method of ultrasonic extraction and the extraction process was optimized. RP-HPLC method is simple, economic and reliable. The separation degree is far more than the demand of quantitative analysis. The method is simple, accurate and reproducible and the experimental results can provide certain reference basis for the quality control of Taxus chinensis var. mairei.

\begin{tabular}{|c|c|c|c|c|c|}
\hline \multicolumn{6}{|c|}{$\begin{array}{c}\text { TABLE-2 } \\
\text { CONTENT OF TWO COMPONENTS IN Taxus chinensis va. mairei collected IN DIFFERENT GROWTH YEAR }(\mathrm{n}=3)\end{array}$} \\
\hline \multirow{2}{*}{ No } & \multirow{2}{*}{ Time (year) } & \multicolumn{2}{|c|}{$\begin{array}{r}\text { Taxol } \\
\end{array}$} & \multicolumn{2}{|c|}{ 10-DAB III } \\
\hline & & Content $\left(\mathrm{mg} \mathrm{g}^{-1}\right)$ & $\mathrm{RSD}(\%)$ & Content $\left(\mathrm{mg} \mathrm{g}^{-1}\right)$ & $\operatorname{RSD}(\%)$ \\
\hline 1 & 1.5 & 0.2892 & 1.45 & 0.5159 & 1.25 \\
\hline 2 & 2 & 0.3276 & 2.06 & 0.6673 & 2.04 \\
\hline 3 & 3 & 0.3930 & 1.33 & 0.9470 & 1.64 \\
\hline 4 & 4 & 0.3375 & 1.92 & 0.6213 & 2.15 \\
\hline 5 & 5 & 0.1539 & 1.67 & 0.1788 & 2.23 \\
\hline
\end{tabular}

\begin{tabular}{cccc|cc}
\hline \multicolumn{9}{c}{ TABLE-3 } \\
CONTENT OF TWO COMPONENTS IN Taxus chinensis va. mairei COLLECTED IN VARIOUS PERIODS $(\mathrm{n}=3)$ \\
\cline { 2 - 6 } No & $\begin{array}{c}\text { Time } \\
\text { (month) }\end{array}$ & Content $\left(\mathrm{mg} \mathrm{g}^{-1}\right)$ & RSD $(\%)$ & Content $\left(\mathrm{mg} \mathrm{g}^{-1}\right)$ & RSD \\
\hline 1 & $2012-03-10$ & 0.2533 & 1.44 & 0.4214 & 1.57 \\
2 & $2012-04-16$ & 0.2940 & 1.92 & 0.4668 & 1.91 \\
3 & $2012-05-14$ & 0.3510 & 2.31 & 0.7229 & 2.28 \\
4 & $2010-06-03$ & 0.3601 & 1.34 & 0.9323 & 1.53 \\
5 & $2010-07-22$ & 0.3365 & 1.57 & 0.6295 & 1.26 \\
6 & $2010-08-13$ & 0.2990 & 1.89 & 0.6028 & 1.06 \\
\hline
\end{tabular}




\section{REFERENCES}

1. W.M. Chen, Acta Pharmacol. Sin., 25, 227 (1990)

2. C.G. Tian and J. Wu, J. Guizhou Normal Univ. (Nat. Sci.), 17, 19 (1999)

3. J. Zhang, X.P. Xu, J. Liu, C.X. Tian and S. Zhou, Chin. J. Pharm. Anal., 16, 28 (2008).

4. C.Z. Sheng, S.F. Wang, Y. Wang and N.N. Wang, Chin. Tradit. Herbal Drugs, 32, 929 (2001).

5. L. Jing-Yu, M. Zhi-da, M. Mizuno, T. Tanaka and M. Iinuma, Phytochemistry, 27, 3674 (1988)

6. Y.Q. Fang, Z.D. Xie and H.Y. Chen, Lishizhen Med. Mater. Med. Res., 23, 2237 (2012).

7. S.Z. Ma and M.B. Wu, Chinese J. New Drugs, 15, 1084 (2006).
8. X. Yang, S.P. Yang, X. Zhang and J. Tan, Di 3 Jun Yi Da Xue Xue Bao, 29, 1886 (2007).

9. Q. Li and X.M. Song, Modern Tradit. Chin. Med., 28, 66 (2008).

10. F. Qian, J. Heze Teachers College, 26, 45 (2004).

11. X.Z. Xu, J. Yanbian Univ. (Nat. Sci.), 24, 42 (1998).

12. M.C. Wani, H.L. Taylor, M.E. Wall, P. Coggon and A.T. Mcphail, J. Am. Chem. Soc., 93, 2325 (1971).

13. G.M. Cragg, S.A. Schepartz, M. Suffness and M.R. Grever, J. Nat. Prod., 56, 1657 (1993).

14. J.R. Su and Z.J. Zhang, Forest Res., 19, 15 (2006)

15. M.C. Bissery, D. Guenad, G. Voegelin and F. Laelle, Cancer Res., 51, 4845 (1991). 